This item was submitted to Loughborough's Research Repository by the author.

Items in Figshare are protected by copyright, with all rights reserved, unless otherwise indicated.

\title{
Introducing speckle noise maps for laser vibrometry
}

PLEASE CITE THE PUBLISHED VERSION

http://dx.doi.org/10.1016/j.optlaseng.2008.06.010

PUBLISHER

(C) Elsevier

VERSION

AM (Accepted Manuscript)

LICENCE

CC BY-NC-ND 4.0

REPOSITORY RECORD

Martin, Peter, and Steve Rothberg. 2019. "Introducing Speckle Noise Maps for Laser Vibrometry". figshare. https://hdl.handle.net/2134/8878. 
This item was submitted to Loughborough's Institutional Repository (https://dspace.lboro.ac.uk/) by the author and is made available under the following Creative Commons Licence conditions.

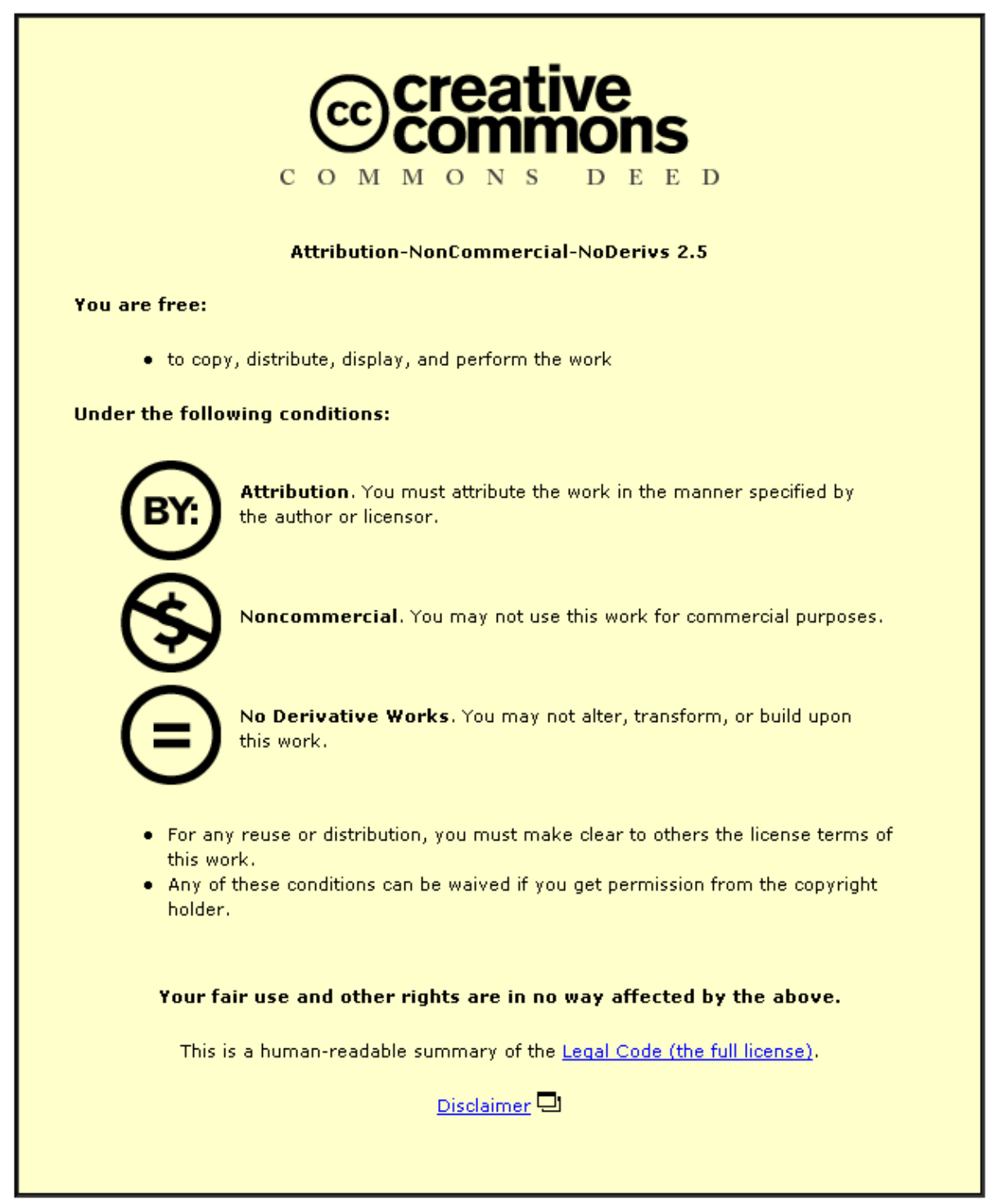

For the full text of this licence, please go to: http://creativecommons.org/licenses/by-nc-nd/2.5/ 


\title{
Introducing speckle noise maps for Laser Vibrometry
}

\author{
Peter Martin \& Steve Rothberg \\ Mechanical \& Manufacturing Engineering \\ Loughborough University, Loughborough. Leics. LE11 3TU. UK \\ p.martin@lboro.ac.uk \\ s.j.rothberg@lboro.ac.uk
}

\begin{abstract}
When coherent light scatters from a surface, which is rough on the scale of the wavelength of the light, a speckle pattern is produced. The Laser Vibrometer measures target vibration velocity in the direction of the incident laser beam and typically samples a region of a speckle pattern on its photodetector. Target motions can cause the speckle pattern to change on the photodetector surface, particularly when target motions are non-normal to the direction of the laser beam. This speckle motion modulates the Doppler signal and adds noise to the demodulated output signal. Periodic target motions can cause the speckle noise to become pseudo-random and produce harmonic peaks, with the same fundamental frequency as the genuine target vibrations, which can be indistinguishable from the genuine target vibrations. Typical speckle noise levels are generally considered to be low-level, but they have not so far been adequately quantified. This paper reports preliminary results quantifying speckle noise levels using controlled experimental configurations incorporating periodic in-plane and tilt target motions. Working with commercial Laser Vibrometers, various target surface finishes and treatments are considered and speckle noise maps are produced for each configuration. For a tilting surface, speckle noise has been quantified at approximately $1 \mu \mathrm{ms}^{-1} / \mathrm{degs}^{-1}$ while, for surfaces with in-plane motion, the sensitivity to speckle noise has been estimated pessimistically at $0.1 \%$ of the in-plane velocity. Ultimately, these speckle noise maps will form a valuable practical resource for the Laser Vibrometer user.
\end{abstract}

\section{Keywords}

Laser Vibrometry; speckle noise; speckle pattern

\section{Introduction}


The Laser Vibrometer is now well established as an effective, non-contact alternative to the use of a traditional contacting vibration transducer. Laser Vibrometers are technically well suited to general application but offer special benefits where certain measurement constraints are imposed, for example by the context, which may demand high frequency operation, high spatial resolution or remote transducer operation, or by the structure itself, which may be hot, sensitive to mass loading or rotating. The technique relies on the detection of a Doppler shift in the frequency of coherent light scattered from a moving surface. When coherent light scatters from a surface that is rough on the scale of the optical wavelength, a speckle pattern is formed. The surface deviations induce phase variations in the component wavelets of the laser beam. Subsequently, the scattered, but still coherent, wavelets interfere constructively and destructively, producing a chaotic distribution of light and dark spots known as a speckle pattern. Surfaces vary in their surface finish, but most surfaces of engineering interest have a roughness comparable with the wavelength of the light. Surfaces with lower roughness produce partially developed speckle patterns within a predominant specular reflection which can divert light away from the receiving aperture and result in low signal amplitude. Surfaces with higher roughness produce fully developed speckle patterns within which the average intensity of the backscattered light is low, again resulting in low signal amplitude. Retro-reflective tape is commonly used to address the issues described above by concentrating the scattered light in a narrow cone centred on the optical axis. Retro-reflective tape is, itself, optically rough and scatter from such a surface still produces a speckle pattern. In all cases, the Laser Vibrometer samples a portion of the speckle pattern and, consequently, the amplitude and phase of the photodetector output result from a phasor summation. The speckle pattern is not of any 
great significance unless it changes dynamically, altering the phasor summation and adding noise to the measurement signal. Non-normal surface motions, in particular, can cause the speckle pattern to translate or boil [1]. The speckle pattern may well show a combination of these two regimes, although one may dominate. In translation, speckles appear to move in space while retaining their size and shape. The boiling regime involves no translation of the speckle but a continuous evolution from one size and shape to another. Both regimes alter the phasor summation on the photo-detector, ultimately causing noise in the output. Speckle motions can add random noise to the Vibrometer output which can become pseudo-random when target motions are periodic. This means that the Vibrometer output spectrum from a measurement on a rough surface vibrating at a single frequency will show speckle noise peaks not only at the same frequency as the genuine vibration but also at many harmonics of it. Therefore the spectral peaks of the pseudo-random speckle noise become coincident with and, more importantly, indistinguishable from genuine vibrations and, for that reason, they are termed pseudo-vibrations [2]. To date, speckle noise has not been adequately quantified and distinguishing genuine motions from pseudo-vibrations relies solely on the experience of the user. The novelty in this paper lies in the quantification of speckle noise which will offer users greater confidence in their data while also showing how beam spot diameters and surface finishes affect speckle noise levels when using commercial Laser Vibrometers.

\subsection{Laser speckle noise}

A laser beam incident on a target surface undergoes a Doppler shift proportional to the component of the surface velocity in the direction of the beam. A frequency shifted reference laser beam is mixed with a backscattered target beam and the resultant 
intensity, $I$, is given by

$$
I=I_{R}+I_{T}+2 \sqrt{I_{R} I_{T}} \cos \left[\omega_{R} t-2 k a(t)+\varphi_{R}-\varphi_{T}\right]
$$

where $I_{R}$ and $I_{T}$ are the reference beam and target beam intensities respectively, $\omega_{R}$ is the reference beam frequency shift, $k$ is the light wavenumber, $a(t)$ is the target surface vibration displacement and $\varphi_{\mathrm{R}}$ and $\varphi_{\mathrm{T}}$ are the phases of the reference and target beam respectively. Demodulation of the resultant signal yields a signal proportional to the target velocity. Non-normal surface motions, in particular, can cause the speckle pattern to become dynamic [2]. This alters the phasor summation and introduces an amplitude and phase modulation to the resultant intensity. These two forms of modulation most likely occur in unison. Phase modulation is a significant contributor to noise, occurring even with adequate signal amplitude. This originates with time dependency in the phase term, $\Delta \varphi(t)$, where $\Delta \varphi(t)=\varphi_{R^{-}} \varphi_{T}(t)$. The measured velocity, $U_{m}$, becomes dependent on the genuine velocity and the additional speckle noise component.

$$
U_{m}=\frac{d a(t)}{d t}-\frac{1}{2 k} \frac{d \Delta \varphi(t)}{d t}
$$

Sudden large changes in Doppler signal amplitude are often accompanied by large changes in phase of the resultant intensity. Insufficient light in backscatter or unfavourable phase additions can result in such inadequate signal amplitude. Furthermore, when the Doppler signal amplitude is very low, dropouts can occur, contributing significant broadband noise [3].

\section{Velocity sensitivity for whole-body target motions}

This paper examines speckle noise for two specific motion types: tilt and in-plane periodic motions. Dedicated test rigs have been built for each motion type. In the 
experiments performed, the genuine velocity in the direction of the laser beam is minimised to reduce measured velocity to a quantity dominated by speckle noise. Periodicity of the surface motions promotes the pseudo-random nature of speckle noise causing spectral peaks at the fundamental vibration frequency and harmonics. To the authors' knowledge, this is the first attempt to quantify speckle noise experimentally and will provide a valuable resource for the vibration engineer.

\subsection{Periodic tilt motion}

A test surface is subjected to a periodic angular vibration, $\dot{\theta}_{z}$, as shown in Figure 1 . The surface angular oscillation was achieved using a lever mechanism attached to a linear shaker. A sinusoidal signal is applied to the linear shaker to generate a periodic tilt of the measurement surface, measured using an accelerometer fixed at a known radial distance from the axis of rotation. The plane of the test surface contains the rotational axis for the tilt motion and the laser beam is aligned normal to the test surface in its equilibrium position such that the genuine velocity in the direction of the incident laser beam is given by [4]:

$$
\frac{d a(t)}{d t} \approx y_{0}\left[\dot{\theta}_{z}\right]
$$

where $y_{0}$ is the y-position of the beam relative to the rotational axis. Experimentally, a micro-positioning device, attached to the Vibrometer, allows minimisation of $y_{0}$ by assessing the measured velocity, temporally and spectrally. The measured velocity can then be assumed to be dominated by speckle noise as follows:

$$
U_{m} \approx \frac{1}{2 k} \frac{\Delta \varphi(t)}{d t}
$$

\subsection{Periodic in-plane motion}


Consideration of in-plane motion requires a pure translation along the z-axis. The measurement surface is attached to a linear rail via a supporting carriage. A linear shaker excites the carriage through a stinger. The stinger is axially aligned with the centre of gravity of the carriage assembly to avoid rotational excitation. A nominally sinusoidal signal is applied to the shaker providing an axial motion of the carriage and measurement surface. An accelerometer is used to measure the axial velocity, $\dot{z}$. Assuming all other motions are successfully made negligible, the genuine velocity in the direction of the incident laser beam is given by [4]:

$$
\frac{d a(t)}{d t} \approx \sin \beta[\dot{z}]
$$

Eq. (5) shows that the angle $\beta$, shown in figure 2 , must be minimised to minimise this genuine component of measured velocity. The procedure requires two Vibrometers. Vibrometer A is used to quantify speckle noise from the test surface. Vibrometer B gives a best estimate of the genuine velocity in the direction of the beam by measuring from a mirrored surface (minimising speckle noise) clamped to the test surface. This mirror was also used to align both beams normal to the surface. For practicality reasons, Vibrometer B was aligned via a second mirror, mounted independently of the vibrating structure. The two beams are made parallel and normal to the mirrored surface. With the beams incident on the mirror, speckle noise is considered to be at a minimum, and the vibration velocity in the direction of the beam can be assessed. Parameter $\beta$ was adjusted for both beams and the measured velocity reassessed. This process was repeated until $\beta$ and, therefore, measured velocity were minimised. The clamped mirror is then repositioned so that it exposes the required measurement surface to the beam of Vibrometer A while remaining as the target surface for the beam of Vibrometer B. The 
measured velocity from Vibrometer A should now be dominated by speckle noise as in Eq. (4).

\section{Vibrometer signal features}

\subsection{Signal dropouts}

Signal dropouts appeared most significant and troublesome on the roughest surface tested $(\operatorname{Ra} 2.39 \mu \mathrm{m})$. This is believed to be principally due to low light levels seen by the Vibrometer. However, dropouts were witnessed in measurements from all surfaces, including a surface treated with retro-reflective tape. Figure 3(a) shows a signal from a tilting surface, with the laser beam aligned such that $y_{0} \neq 0$, exhibiting broadband speckle noise and also significant periodic dropouts. With slight manipulation of the beam position relative to the surface, so the sampled region of the speckle pattern changes slightly, the signal dropouts seen in Figure 3(a) can progress into an unusable signal with high apparent velocities, shown in Figure 3(b). With modest manipulation of the beam location, the signal dropouts can also be made to disappear to enable high quality measurements.

Figure 4 displays a closer analysis of the dropouts seen in Figure 3(a), clearly showing a significant reduction of the amplitude in the Doppler signal prior to the dropout. The generation of such impulses has also been seen in simulations, [3], which show large phase changes occurring alongside large changes in Doppler signal amplitudes. These impulses contain significant energy and can raise the broadband noise to levels where a useful measurement becomes unattainable. The data presented in Figure 4 is from a target treated with retro-reflective tape. Therefore, the use of retro-reflective tape, which does increase the intensity of the light in backscatter, does not guarantee a signal clear 
of dropouts. However, the signal can usually be cleared of such dropouts by slight transferral of the measurement location on the test surface. This improves the signal by sampling a region of the speckle pattern from which higher Doppler signal amplitude is maintained without any sudden changes in that amplitude.

\subsection{Speckle noise}

Figure 5 shows Vibrometer outputs from a measurement on a tilting target with the incident beam positioned such that the measured velocity is minimised i.e. $y_{0} \approx 0$. The apparent velocities, labelled as 'Speckle noise', were acquired with vibration frequencies of $10 \mathrm{~Hz}$ and $30 \mathrm{~Hz}$. In particular, the measurements at each frequency were sampling similar (nominally identical) dynamic speckle patterns for two periods of sinusoidal motion with equal displacement amplitudes. The secondary plot shows the resulting phase in radians, calculated from the integral of the output signal. The distinguishing peaks, encircled in Figure 5, are prominent features in speckle noise. They appear to negate and reflect about an instant in time corresponding to the extreme of the displacement cycle. This is due to the phase change that can be seen in the speckle noise integral. As the target moves from one extreme of its displacement cycle to the other extreme, the phase of the resultant Doppler signal changes in a continuous sequence as the sampled speckle pattern changes. In the second half of the displacement cycle, the phase of the resultant intensity passes through the same sequence of phases as the first half of the cycle but in reverse. Consequently the changes in phase have the same magnitude in the second half of the cycle but have an opposite sign. Increasing the vibration frequency while maintaining the angular surface displacement amplitude shows an increased level of speckle noise. The speckle noise integral plots show that the phase change remains consistent regardless of the vibration frequency. Increasing the 
vibration frequency means the phase change occurs more quickly and consequently speckle noise levels increase in direct proportion to vibration frequency. This is typical temporal data from which speckle noise is quantified in this paper.

Closer assessment of these peaks synchronised with the Doppler signal is shown in Figure 6. The Doppler signal amplitude shows a significant variation prior to the peak shown in the Vibrometer output. This is the result of a significant change in the summation of speckles on the photodetector, leading to both the amplitude modulation shown in the Doppler signal and the phase modulation appearing in the demodulated output. This shows how, even when the Doppler signal amplitude remains adequate and accurate demodulation is attainable, speckle changes still cause phase modulations and speckle noise appears in the output from the Vibrometer. What differentiates the signals seen in Figure 6 from those in Figure 4 are the level of amplitude modulation and the resulting speckle noise amplitude.

\section{Speckle noise map for commercial instruments}

Speckle noise is quantified for two commercial Vibrometers, a Polytec OFV302 translational Vibrometer head (incident beam spot diameter of $100 \mu \mathrm{m}$ ) with OFV3000 controller and the Polytec OFV400 rotational Vibrometer head (incident beam spot diameter of $700 \mu \mathrm{m}$ ) with OFV4000 controller used as a single point translational Vibrometer. With target beams aligned as described in section 2, speckle noise is quantified for various surface finishes with signals for which every effort has been made to exclude signal dropouts such as those shown in Figure 3. At each frequency, vibration displacement amplitude was held nominally constant. Visual observations of speckle and speckle pattern motions qualitatively aid consideration of speckle noise and 
Section 3.2 accompanied by Figure 5 described how vibration frequency affects speckle noise. Speckle noise is therefore presented as a normalised ratio versus order for $10 \mathrm{~Hz}$, $20 \mathrm{~Hz}$ and $30 \mathrm{~Hz}$ vibration frequencies. Data in this form can then be extrapolated to any vibration frequency and amplitude.

\subsection{Periodic tilting target surface motion}

For the tilting motion, results have been taken from surfaces with roughnesses of Ra $0.19 \mu \mathrm{m}(\approx 0.3 \lambda$, where $\lambda=$ laser wavelength $), \operatorname{Ra} 2.39 \mu \mathrm{m}(\approx 4 \lambda)$ and also a surface treated with retro-reflective tape. Excitation frequencies ranged from $10 \mathrm{~Hz}$ to $30 \mathrm{~Hz}$ with nominally constant angular displacement of $0.16^{\circ} \mathrm{rms}$ for each experimental configuration.

\subsubsection{Speckle motion observations}

The speckle pattern motions with the beam positioned on the rotational axis, $y_{0} \approx 0$, can be observed in Figures 7 and 8. The leftmost frames in Figures 7(a-c) show the speckle patterns for a beam spot diameter of $100 \mu \mathrm{m}$. Large speckles are the result of the small beam spot size. The subsequent frames, magnified from the highlighted rectangular region in the leftmost frame, show the speckle pattern motion following incremental angular displacements of approximately $0.15^{\circ}$ between frames. The circles track specific regions in the speckle pattern to show how speckles change through these incremental displacements. The total translation of the circled region is approximately equal to the diameter of the collecting aperture on the Vibrometer. The Vibrometer aperture can be seen as the static high intensity region (due to the outgoing beam) in the centre of the first image encircled by a reticle $20 \mathrm{~mm}$ in diameter. Figure 7 (a) shows the speckle pattern produced from a surface with roughness Ra $0.19 \mu \mathrm{m}$. The vertical 
concentration of the scatter is perpendicular to the direction of the surface corrugations produced by polishing. A high intensity region, which is seen just above the aperture on Figure 7(a) is formed by specular reflection from the polished surface. The circled regions indicate translational speckle motion with negligible evolution. Figure 7(b) shows the speckle patterns produced from a surface treated with retro-reflective tape. There is a translation and also some evolution of the speckle during the target motion. Retro-reflective tape is coated in micro-scale glass beads, approximately $50 \mu \mathrm{m}$ diameter. For a small spot diameter, the number of glass beads contributing to the speckle pattern is small and the target surface motion changes the population of scatterers significantly, causing speckle evolution. Figure 7(c) displays the speckle pattern produced from a surface with roughness Ra $2.39 \mu \mathrm{m}$. There is a concentration of the speckle intensity in a vertical direction perpendicular to the machining finish direction. The speckle motion shows considerable translation and negligible evolution over the five frames, as for the surface in Figure 7(a). Translation is the expected speckle motion for a surface tilt and so the degree of evolution for the surface treated with retro-reflective tape is noteworthy.

Figures $8(\mathrm{a}-\mathrm{c})$ show the speckle patterns for a beam spot diameter of $700 \mu \mathrm{m}$ from which much smaller speckles result. Figure 8(a) shows the speckle pattern and speckle motions for a surface of roughness $\mathrm{Ra} 0.19 \mu \mathrm{m}$. The speckle motion exhibits significant translation and negligible evolution. Figure 8(b) shows the speckle pattern and speckle motion for a surface treated with retro-reflective tape. In this case, the circled regions continue to show evolution of the speckle pattern but there is now also a much more distinct translation. Figure 8(c) shows the speckle pattern and speckle motion for a surface with roughness $\mathrm{Ra} 2.39 \mu \mathrm{m}$. The speckle motion is dominated by translation. 
Speckle boiling motion is emphasised when there is a significant change to the population of scatterers. A change to the population of scatterers occurs when there is a change to the illuminated area on the surface. In this experiment, with the beam positioned on the rotational axis, i.e. $y_{0}=0$, the beam spot elongates during the angular oscillation, changing the population of scatterers but the dominance of speckle translation, seen in figures 7 and 8 , indicates this change to be insignificant.

\subsubsection{Speckle noise in measurements on a tilting target surface}

The following results quantify, pessimistically, the first 50 orders of speckle noise by normalising the Vibrometer output against the angular velocity at the fundamental frequency. Each data series takes 15 averages from 3 separate locations on the surface, thereby quantifying speckle noise levels using uncorrelated speckle patterns. As shown in section 4.1.1, speckle motions are dominated by the translation regime. Figure 9 shows 50 orders of the mean normalised speckle noise, $+/$ - one standard deviation, for measurements from a surface with roughness $\mathrm{Ra} 0.19 \mu \mathrm{m}$ for vibrations with fundamental frequencies of $10 \mathrm{~Hz}, 20 \mathrm{~Hz}$ and $30 \mathrm{~Hz}$. Presenting speckle noise normalised against the angular velocity at the fundamental frequency versus the order removes the effect of the oscillation frequency and also shows how speckle noise is dependent on the rate, $d t$ in Eq. (2), of the change in phase. This presentation allows speckle noise to be conveniently quantified for each surface finish at any frequency of oscillation and figures 10 and 11 show the mean speckle noise levels with $+/$ - one standard deviation for each surface finish. Table 1 shows the sensitivity to speckle noise for each surface. While the differences are not very large, the surface with roughness Ra $2.39 \mu \mathrm{m}$ 
produces the lowest speckle noise levels and the surface treated with retro-reflective tape produces the highest. This is highlighted in Figure 11 which shows slightly higher speckle noise levels for the surface treated with retro-reflective tape particularly at higher harmonic orders. This may be as a result of the additional evolutionary speckle motions, described in section 4.1.1, causing more rapid changes in the phase of the resultant Doppler signal.

With a beam spot diameter of $100 \mu \mathrm{m}$, speckle noise levels are much reduced at higher order harmonics. This is evident in Figure 11 where the noise levels reduce significantly and become indistinguishable from the baseline noise level. Comparing Figures 10 and 11 , the difference in speckle noise levels is attributed to the variation in spot size. A smaller laser spot diameter on the surface increases the size of the speckle [5], evident in Figures 7 and 8. Larger speckles produce phase variations which occur over a greater time and therefore reduce higher frequency noise components.

Table 1 shows speckle noise sensitivity in two different formats. The first is as a mean and standard deviation calculated over the first ten orders. Ten orders is chosen as a typical bandwidth over which the vibration engineer is likely to be interested. A range of approximately 0.1 to $1 \mu \mathrm{ms}^{-1} / \mathrm{degs}^{-1}$ is found. The second format is the total level, calculated as a ratio of the total $\mathrm{rms}$ of the apparent velocity across 50 orders to the rms of the angular velocity at the fundamental frequency, with values in the range 0.3 to 3.3 $\mu \mathrm{ms}^{-1} / \mathrm{degs}^{-1}$. Figure 12 presents the first-ever mapping, to the authors' knowledge, of speckle noise for Laser Vibrometers. The levels roll off as the harmonic order increases and this roll off is large for the beam diameter of $100 \mu \mathrm{m}$. These results, from a tilting target, suggest focussing the beam whenever possible to reduce speckle noise. This is in 
fundamental accord with Drain [6], who remarked 'increasing the spot size...increases sensitivity to tilt of the surface'. It is also consistent with conventional wisdom on minimising frequency broadenings by minimising the range of Doppler shifts across the finite extent of the incident laser beam.

\subsection{In-plane target surface motion}

For in-plane motion, measurements were made on a surface with a roughness of $\mathrm{Ra}$ $0.0618 \mu \mathrm{m}(\approx 0.1 \lambda)$ and also on a surface treated with retro-reflective tape. A sinusoidal displacement of $0.25 \mathrm{~mm} \mathrm{rms}$ is used with fundamental frequencies of $10 \mathrm{~Hz}, 20 \mathrm{~Hz}$ and $30 \mathrm{~Hz}$.

\subsubsection{Speckle motion observations}

The speckle behaviour from an in-plane motion is dominated by evolution. This is due to the alteration in the population of scatterers as the surface translates across the incident beam.

The frames shown in Figures 13(a \& b) display the speckle changes occurring due to incremental in-plane linear displacements of approximately $0.12 \mathrm{~mm}$, with a beam diameter of $700 \mu \mathrm{m}$. Figure 13(a) shows the speckle pattern when the target beam is incident on retro-reflective tape and figure 13(b) for when the beam is incident on a surface with roughness Ra $0.0618 \mu \mathrm{m}$. Evident within the circled regions is the progressive evolution of the speckle. Further observation of these speckle patterns shows significant evolution of speckles, but also slight speckle translation. Figures 14(a $\&$ b) display the speckle patterns and their motions for the beam diameter of $100 \mu \mathrm{m}$. The circled regions show negligible translation and a distinct evolution which appears 
greater than that seen in Figures $13(\mathrm{a} \& \mathrm{~b}$ ). For the smaller beam, a given displacement of the target surface results in a greater change in the population of scatterers which decorrelates the speckle pattern more quickly.

\subsubsection{Speckle noise in measurements on a target surface moving in-plane}

Speckle noise is quantified over 50 orders and is pessimistic in its evaluation. The minimum, genuine out-of-plane velocity achievable was estimated to be $1 / 1000$ of $\dot{z}$. Therefore, speckle noise is not necessarily the dominant component in the measured velocity, particularly at lower harmonic orders. Figure 15 shows the apparent velocity normalised against the in-plane velocity at the fundamental frequency when using a beam diameter of $100 \mu \mathrm{m}$. As the harmonic order increases, the spectral amplitudes of the data from retro-reflective tape and the Ra $0.0618 \mu \mathrm{m}$ surface become distinguishable from the genuine velocity and speckle noise can be assumed to be the dominant contribution to the apparent velocity. The Ra $0.0618 \mu \mathrm{m}$ surface shows lower speckle noise levels, most noticeable at higher harmonic orders. This is thought to be as a result of the specular element in the backscattered light reducing the overall phase variation. Figure 16 shows the measured velocity normalised against the in-plane velocity at the fundamental frequency when using a beam diameter of $700 \mu \mathrm{m}$. The speckle noise amplitudes are lower, particularly at high orders, in comparison to those produced when using the smaller beam diameter. However, the estimated speckle noise levels here are very similar to the measured, genuine velocity at all harmonic orders. It is therefore believed that the measured velocity with beam diameter of $700 \mu \mathrm{m}$ is dominated by the genuine velocity. Taking these values for speckle noise is very pessimistic but the best estimate currently achievable. 
Figure 17 presents preliminary estimates of speckle noise for a target surface with an inplane motion. Table 2 shows speckle noise estimated for each surface. Speckle noise sensitivity (mean and standard deviation) is calculated over the first ten orders and is approximated, pessimistically, as $0.1 \%$. The total level is calculated as the ratio of the total rms of the apparent velocity across 50 orders against the rms of the in-plane velocity at the fundamental frequency, indicating a sensitivity to speckle noise at approximately $0.3 \%$. Although components of genuine velocity remain present in the data presented above, there is clear, quantitative evidence for the use of a larger beam diameter when in-plane components of velocity are present. The increased beam size decreases the rate of change of the population of scatterers reducing speckle noise, particularly at higher orders.

\section{Conclusions}

This paper presents the first experimental estimates of speckle noise in commercial Laser Vibrometers for periodic tilting and in-plane surface motions. The data also indicate how surface finish and beam spot diameter affect speckle noise.

For tilting target motion, visual observation of speckle motions showed domination of speckle translation. Reducing the beam spot diameter resulted in larger speckles and slower phase variations, consequently reducing higher order frequency components of speckle noise. A focussed beam is therefore recommended for a surface in tilt. The mean plus one standard deviation for the first ten orders of speckle noise was shown to be a maximum of $1 \mu \mathrm{ms}^{-1} / \operatorname{degs}^{-1}$.

In-plane surface motion produces significant changes in the illuminated population of scatterers and observations of speckle motion reveal domination of boiling. For this 
motion, reducing the beam spot diameter promoted more rapid speckle pattern changes, increasing higher order components of speckle noise. Larger beam size is therefore recommended to minimise speckle noise. A polished surface $(\mathrm{Ra} \approx 0.1 \lambda)$ appears to reduce high harmonic orders of speckle noise because strong specular reflection reduces the overall phase modulation in the resultant Doppler signal. The mean plus one standard deviation of speckle noise, over the first ten orders, for an in-plane target motion has been estimated as $0.1 \%$ of the in-plane velocity.

\section{References}

[1] N Takai, T Iwai and T Asakura. Correlation distance of dynamic speckles, Journal of Applied Optics, 22(1) 170-177, January 1983

[2] SJ Rothberg, JR Baker \& NA Halliwell, Laser Vibrometry: Pseudo-vibrations, Journal of Sound and Vibration (1989) 135(3), 516-522

[3] SJ Rothberg, Numerical simulation of speckle noise in Laser Vibrometry, Journal of Applied Optics, 45(19), 4523-4533, July 2006

[4] J.R.Bell, S.J.Rothberg, Laser Vibrometers and contacting transducers, target rotation and six degree-of-freedom vibration: What do we really measure?, Journal of Sound and Vibration 237(2), 245-261, March 2000

[5] JW Goodman, Statistical Properties of Laser Speckle Patterns, Laser Speckle and Related Phenomena, J.C. Dainty, Springer-Verlag. 9-74. 1984

[6] LE Drain, The Laser Doppler Technique, Wiley, 222-225, 1980 


\begin{tabular}{|c|c|c|c|c|}
\hline \multirow{2}{*}{$\begin{array}{l}\text { Beam spot } \\
\text { diameter } \\
\text { (Vibrometer } \\
\text { Head) }\end{array}$} & \multirow{2}{*}{$\begin{array}{l}\text { Surface finish } \\
(\operatorname{Ra} \mu \mathrm{m})\end{array}$} & \multicolumn{3}{|c|}{$\begin{array}{l}\text { Speckle } \\
\text { Apparent velocity per unit angular velocity, } \mu \mathrm{ms}^{-1} / \mathrm{degs}^{-1}\end{array}$} \\
\hline & & $\begin{array}{l}\text { Mean level by order } \\
\text { (first } 10 \text { orders) }\end{array}$ & $\begin{array}{l}\text { Standard deviation } \\
\text { (first } 10 \text { orders) }\end{array}$ & $\begin{array}{l}\text { Total level across } \\
50 \text { orders }\end{array}$ \\
\hline \multirow{3}{*}{$\begin{array}{l}100 \mu \mathrm{m} \\
(\mathrm{OFV} 302)\end{array}$} & 0.19 & 0.13 & 0.13 & 0.46 \\
\hline & 2.39 & 0.075 & 0.057 & 0.32 \\
\hline & retro-reflective tape & 0.14 & 0.16 & 0.51 \\
\hline \multirow{3}{*}{$\begin{array}{l}700 \mu \mathrm{m} \\
\text { (OFV400) }\end{array}$} & 0.19 & 0.64 & 0.39 & 3.3 \\
\hline & 2.39 & 0.33 & 0.22 & 1.4 \\
\hline & retro-reflective tape & 0.31 & 0.33 & 1.7 \\
\hline
\end{tabular}

Table 1: Speckle noise mean, standard deviation and rms levels

(OFV302 using retro-reflective tape, anomalous results excluded at order numbers 14, 15 \& 16)

\begin{tabular}{|c|c|c|c|c|}
\hline \multirow{2}{*}{$\begin{array}{l}\text { Beam spot } \\
\text { diameter } \\
\text { (Vibrometer } \\
\text { Head) }\end{array}$} & \multirow{2}{*}{$\begin{array}{l}\text { Surface finish } \\
(\operatorname{Ra} \mu \mathrm{m})\end{array}$} & \multicolumn{3}{|c|}{$\begin{array}{l}\text { Speckle } \quad \text { noise } \\
\text { Apparent velocity per unit in-plane velocity, } \%\end{array}$} \\
\hline & & $\begin{array}{l}\text { Mean level by order } \\
\text { (first } 10 \text { orders) }\end{array}$ & $\begin{array}{l}\text { Standard deviation } \\
\text { (first } 10 \text { orders) }\end{array}$ & $\begin{array}{l}\text { Total level across } \\
50 \text { orders }\end{array}$ \\
\hline \multirow{2}{*}{$\begin{array}{l}100 \mu \mathrm{m} \\
(\mathrm{OFV} 302)\end{array}$} & 0.0618 & 0.044 & 0.026 & 0.19 \\
\hline & retro-reflective tape & 0.064 & 0.040 & 0.28 \\
\hline \multirow{2}{*}{$\begin{array}{l}700 \mu \mathrm{m} \\
(\mathrm{OFV} 400)\end{array}$} & 0.0618 & 0.043 & 0.030 & 0.18 \\
\hline & retro-reflective tape & 0.037 & 0.024 & 0.16 \\
\hline
\end{tabular}

Table 2: Speckle noise mean, standard deviation and rms levels 


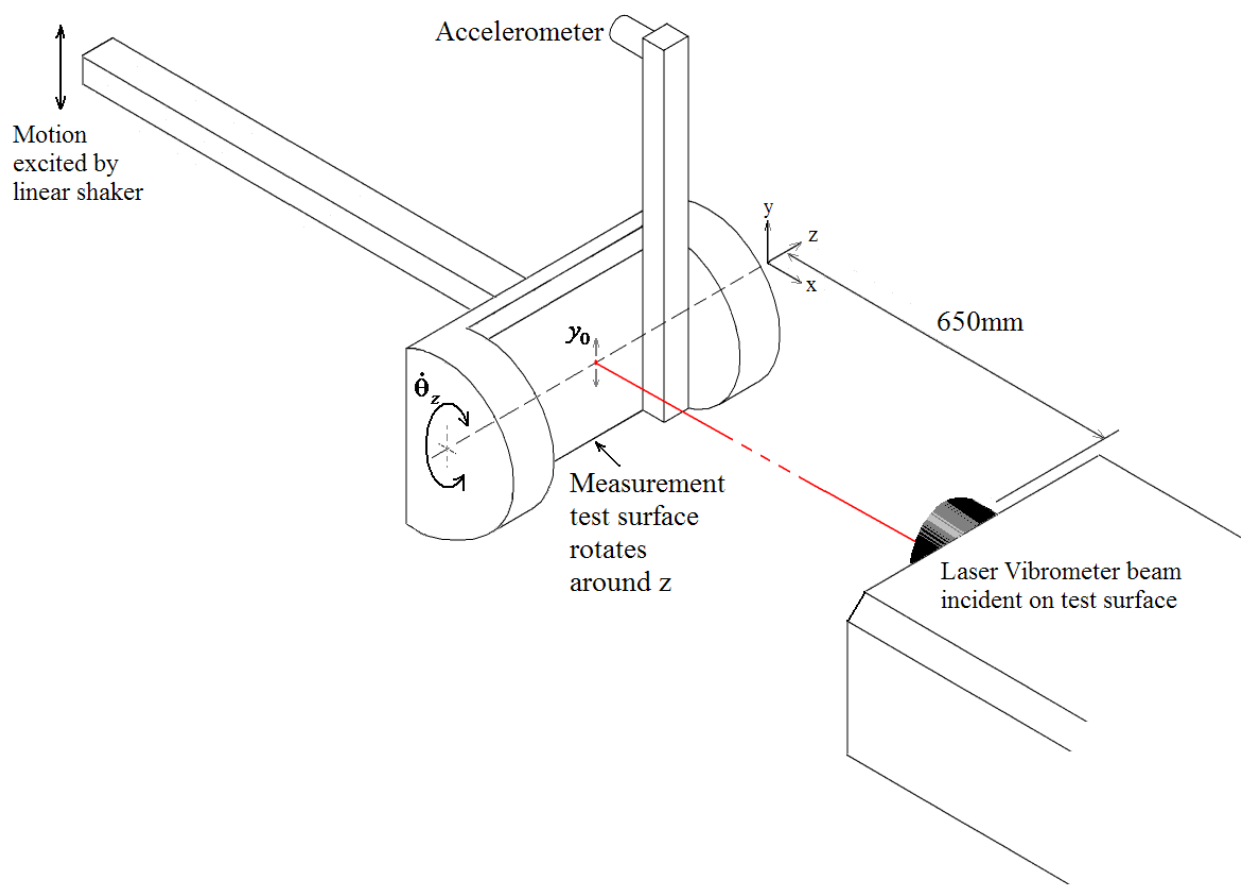

Figure 1 - Schematic of tilt experimental configuration

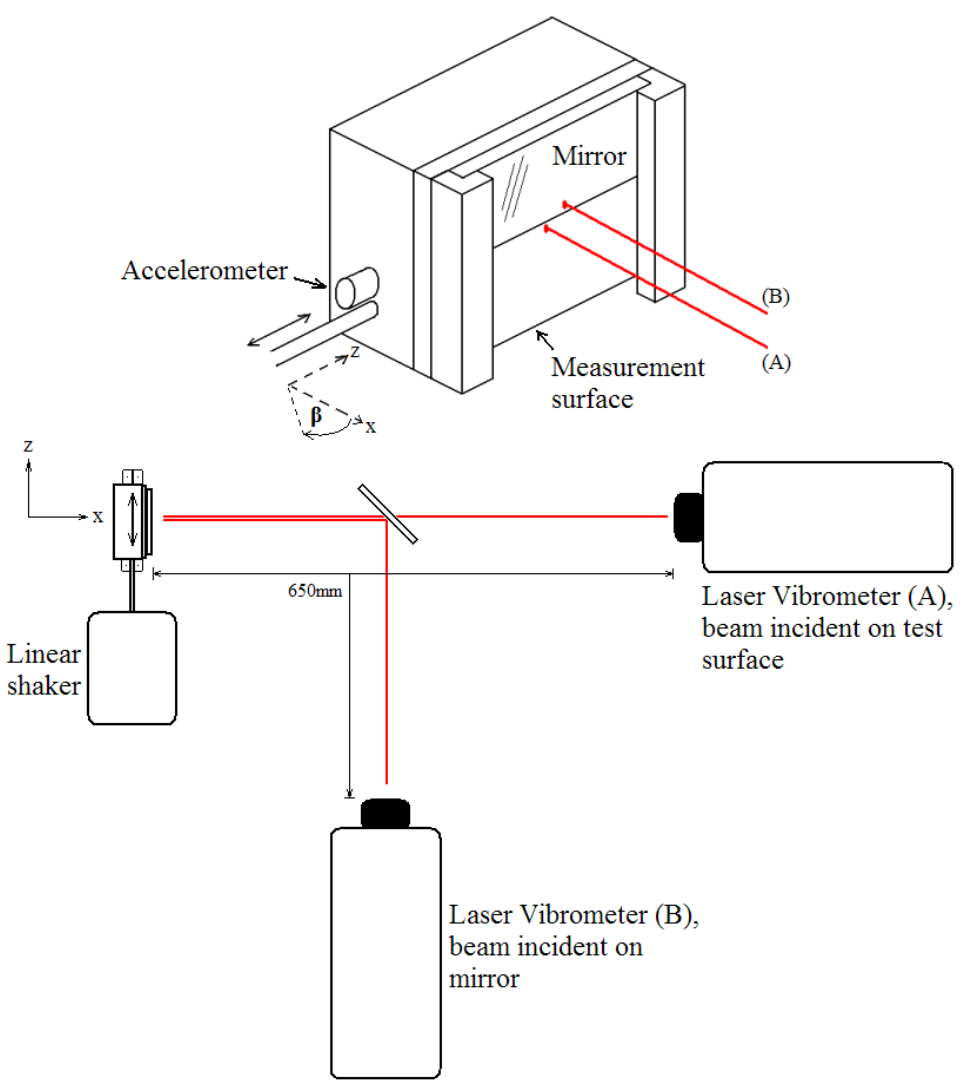

Figure 2 - Schematic of in-plane speckle sensitivity measurement 


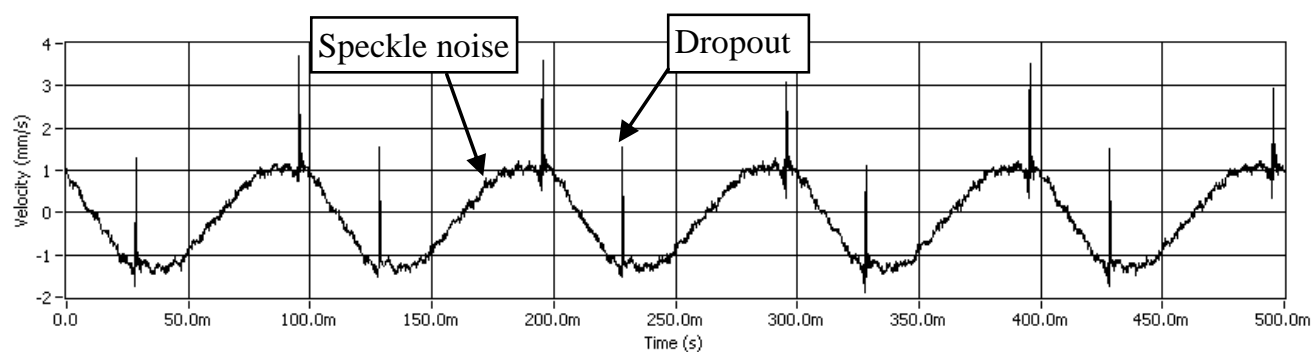

(a)

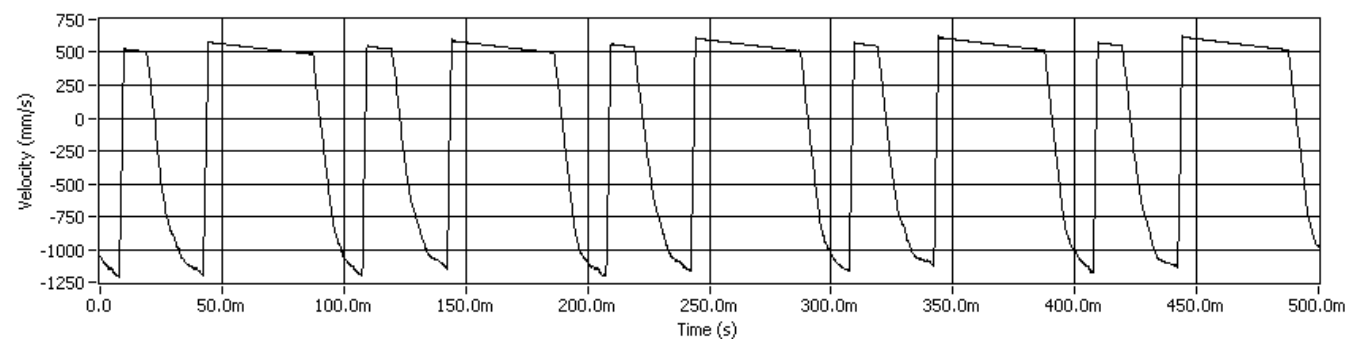

(b)

Figure 3-Vibrometer outputs showing (a) periodic dropouts (b) unusable signal. Tilting surface with roughness Ra2.39 $\mu \mathrm{m}$ oscillating at $10 \mathrm{~Hz}$
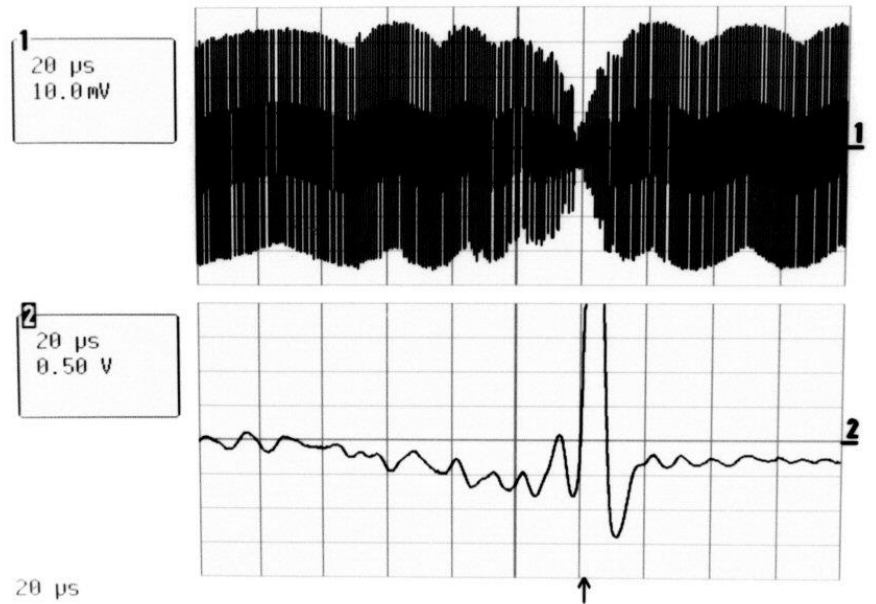

$118 \mathrm{mV} \quad \mathrm{AC}$

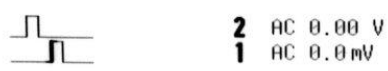

$180 \mathrm{MS} / \mathrm{s}$

口 STOPPEI

Figure 4- Doppler signal (top) synchronised with the Vibrometer output signal (bottom) showing a typical signal dropout from a tilting surface treated with retro-reflective tape oscillating at $30 \mathrm{~Hz}$ 
on $200 \mu$ s timescale

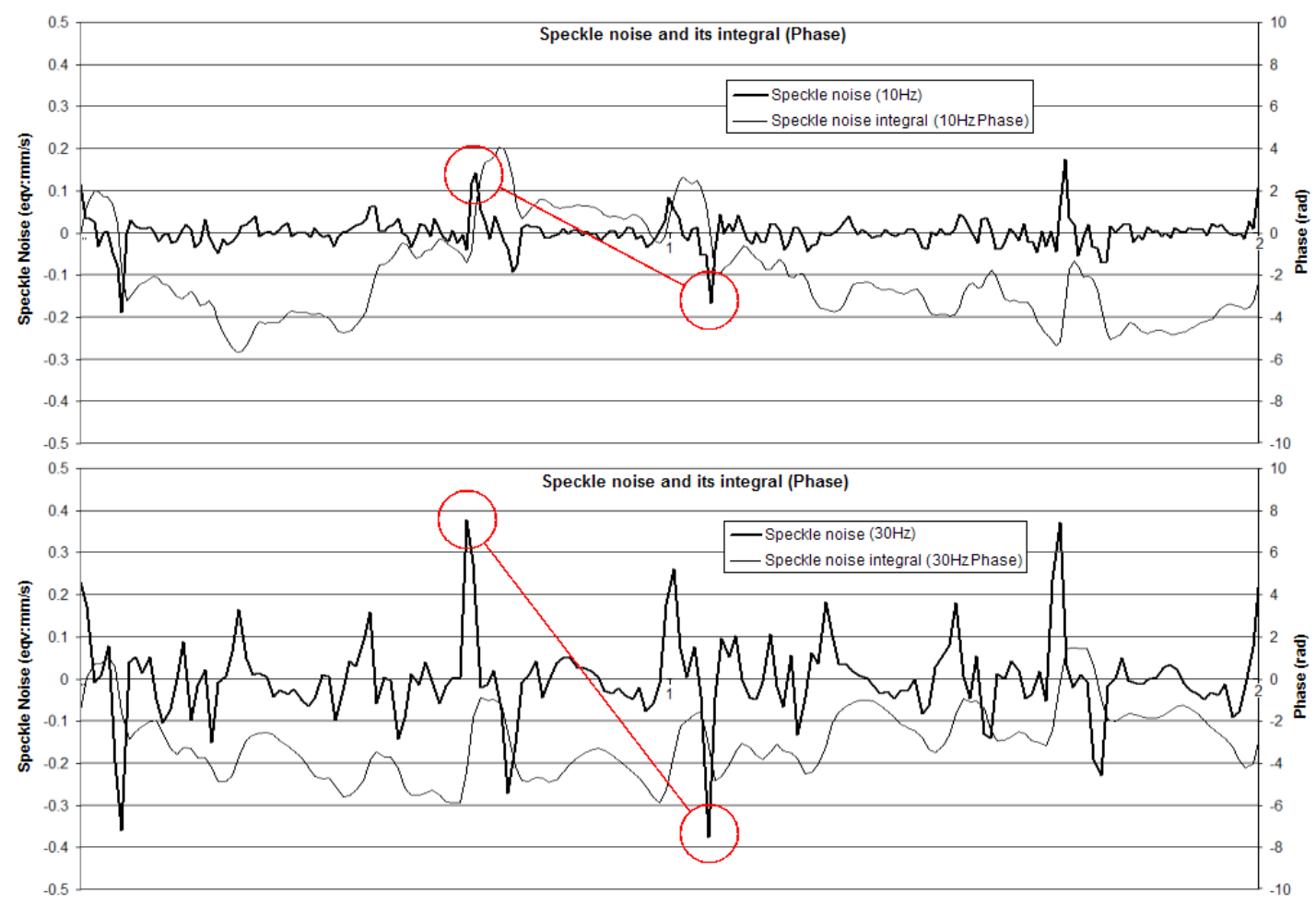

Figure 5 - Speckle noise represented as an apparent velocity in $\mathrm{mm} / \mathrm{s}$ and phase in radians from two measurements sampling similar speckle patterns from a tilting target surface treated with retro-reflective tape oscillating at (a) $10 \mathrm{~Hz}$ and (b) $30 \mathrm{~Hz}$
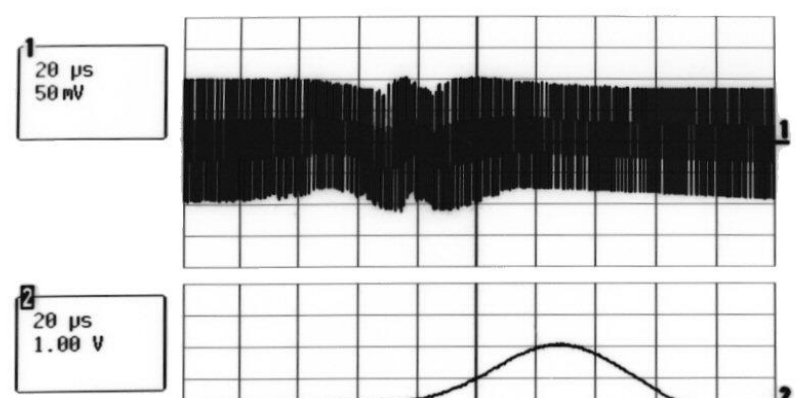

$20 \mu \mathrm{s}$

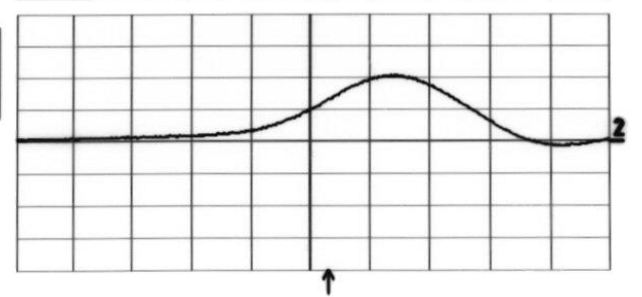

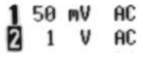

$\Omega$

$2 \mathrm{AC} 1.48 \mathrm{~V}$

$100 \mathrm{MS} / \mathrm{s}$

口 STOPPED

Figure 6 -Doppler Signal (top) synchronised with the Vibrometer output (bottom) showing typical speckle noise peak. Tilting surface treated with retro-reflective tape oscillating at $30 \mathrm{~Hz}$ on a $200 \mu$ s timescale. 


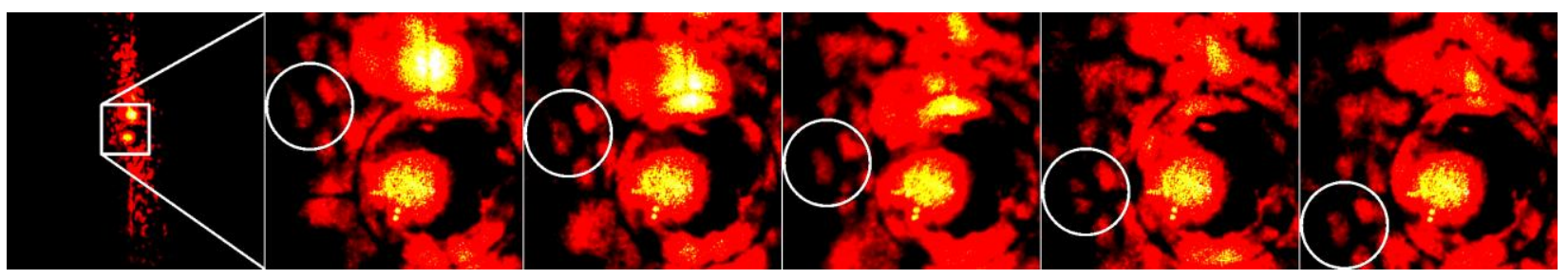

(a)

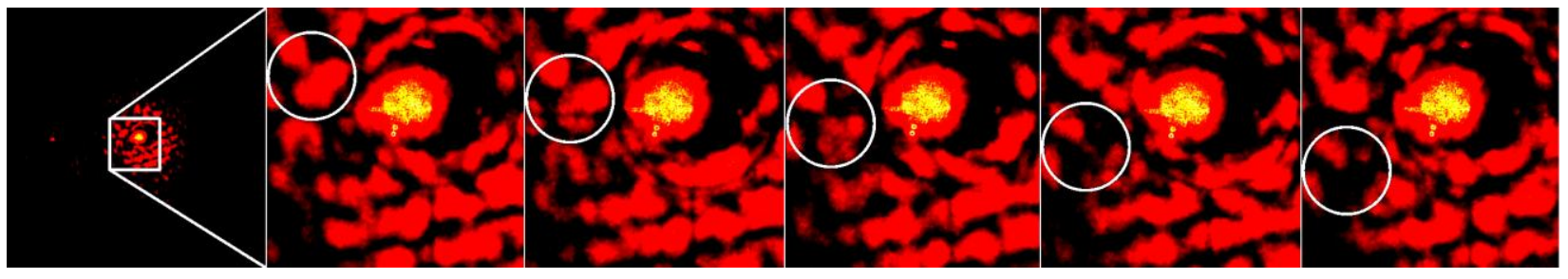

(b)

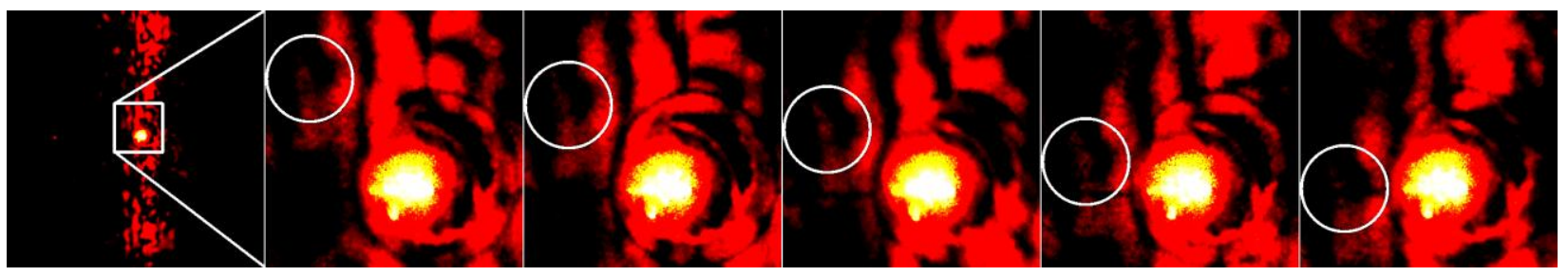

(c)

Figure 7 Speckle pattern and the motions from (a) metallic surface with roughness Ra $0.19 \mu \mathrm{m}$, (b) retroreflective tape, (c) metallic surface with roughness Ra $2.39 \mu \mathrm{m}$ using a beam spot diameter $100 \mu \mathrm{m}$ 


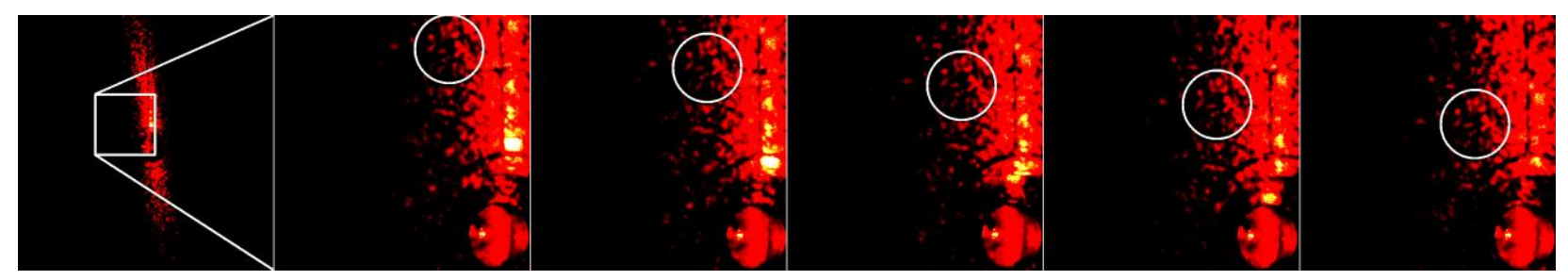

(a)

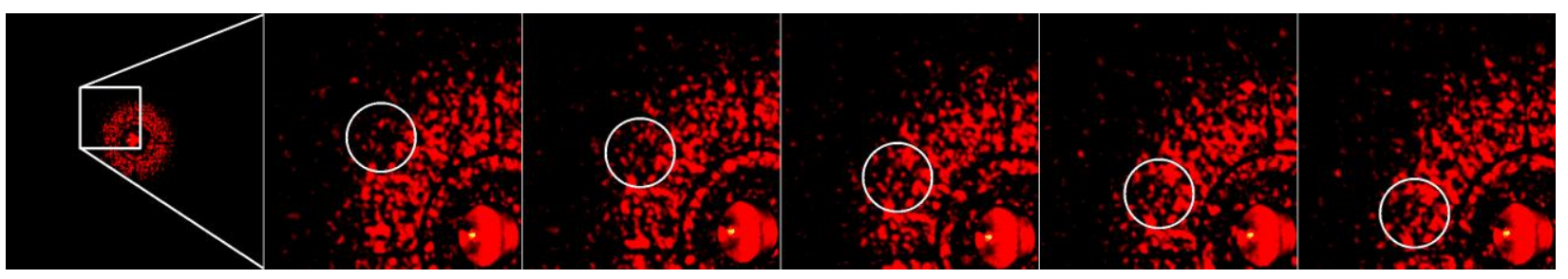

(b)

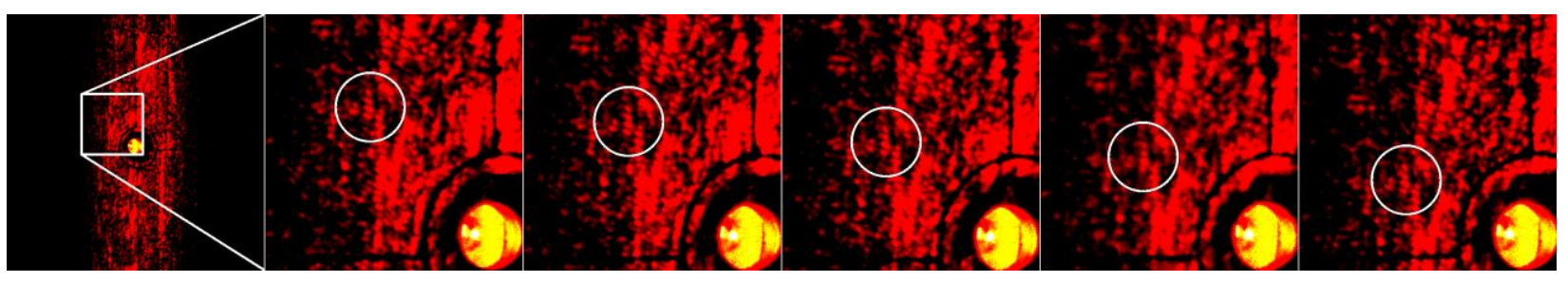

(c)

Figure 8 - Speckle pattern and the motions from (a) metallic surface with roughness Ra $0.19 \mu \mathrm{m}$, (b) retro-reflective tape $\&$ (c) metallic surface with roughness Ra $2.39 \mu \mathrm{m}$ using a beam spot diameter $700 \mu \mathrm{m}$

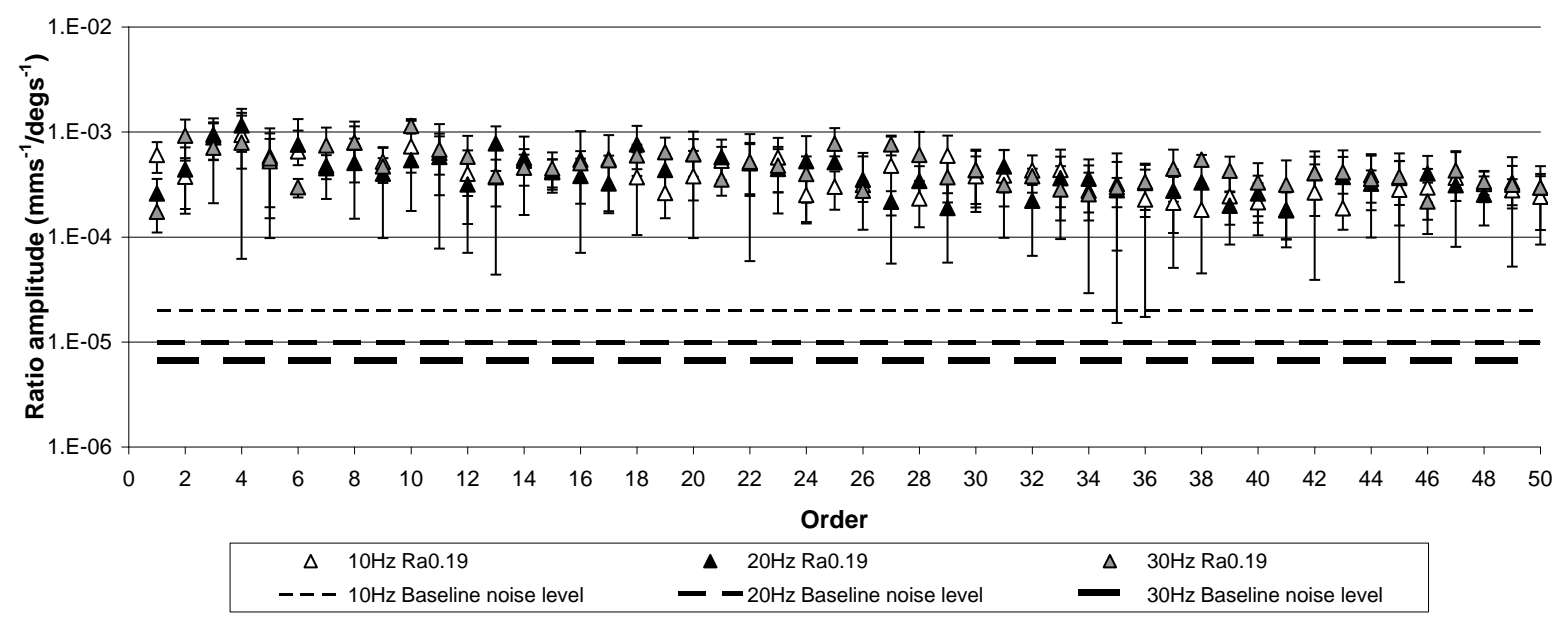

Figure 9 - Speckle noise normalised against the angular velocity $\left(\mathrm{mms}^{-1} / \mathrm{degs}^{-1}\right)$ at the fundamental frequency using a beam spot diameter $700 \mu \mathrm{m}$ on a surface with roughness Ra $0.19 \mu \mathrm{m}$. 


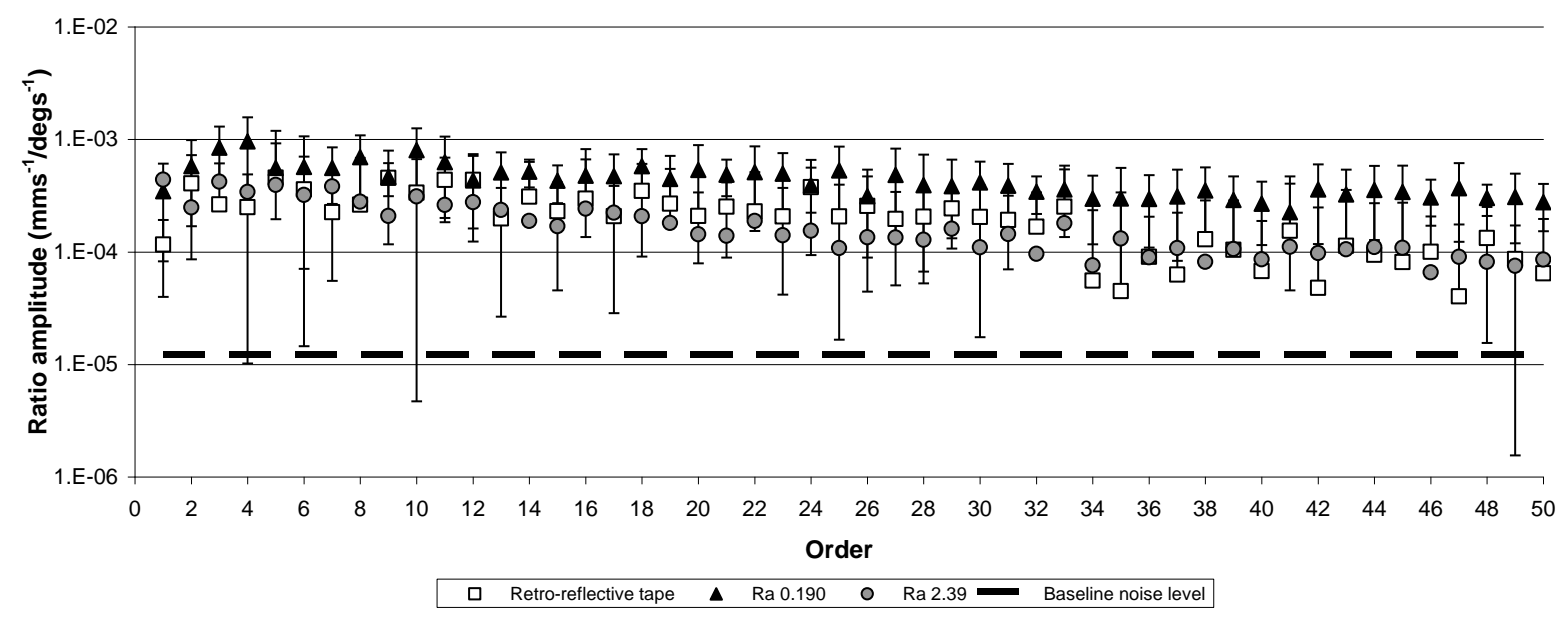

Figure 10 - Speckle noise normalised against the angular velocity $\left(\mathrm{mms}^{-1} / \mathrm{degs}^{-1}\right)$ at the fundamental frequency using a beam spot diameter $700 \mu \mathrm{m}$

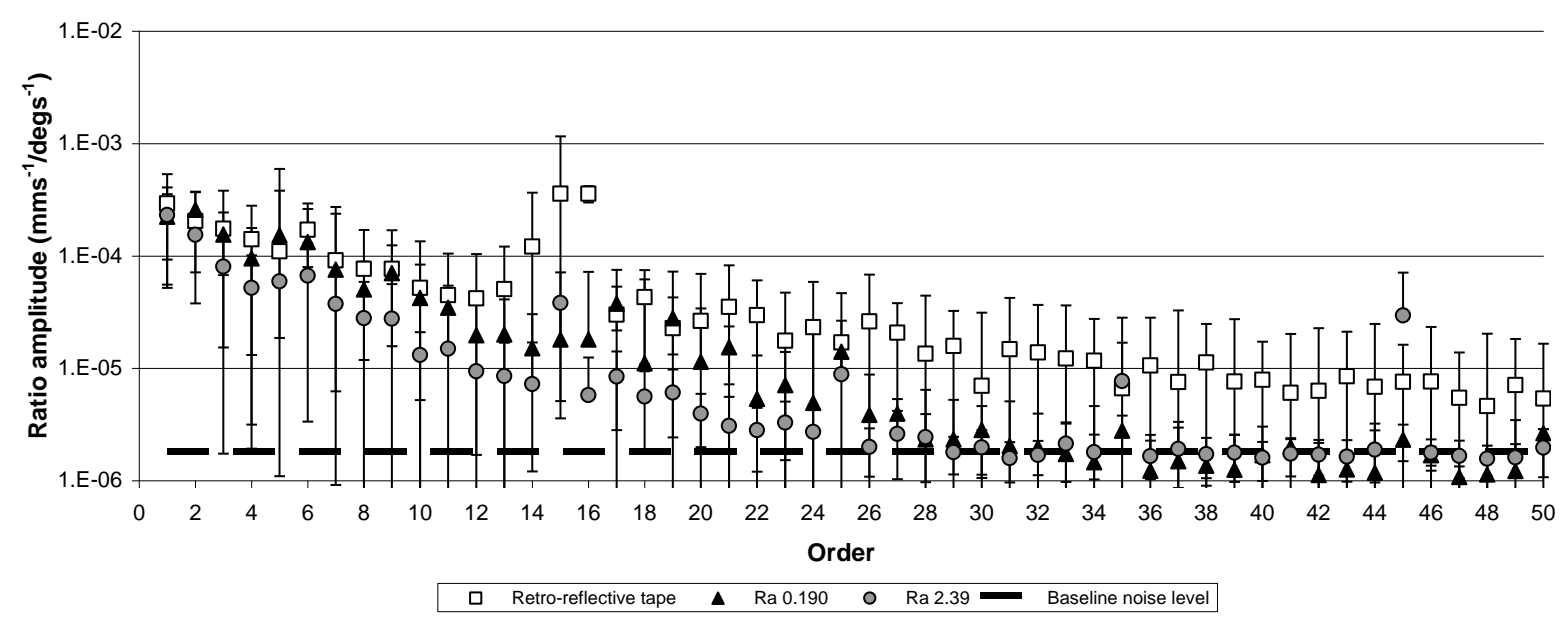

Figure 11 - Speckle noise normalised against the angular velocity $\left(\mathrm{mms}^{-1} / \mathrm{degs}^{-1}\right)$ at the fundamental frequency using a beam spot diameter $100 \mu \mathrm{m}$ 


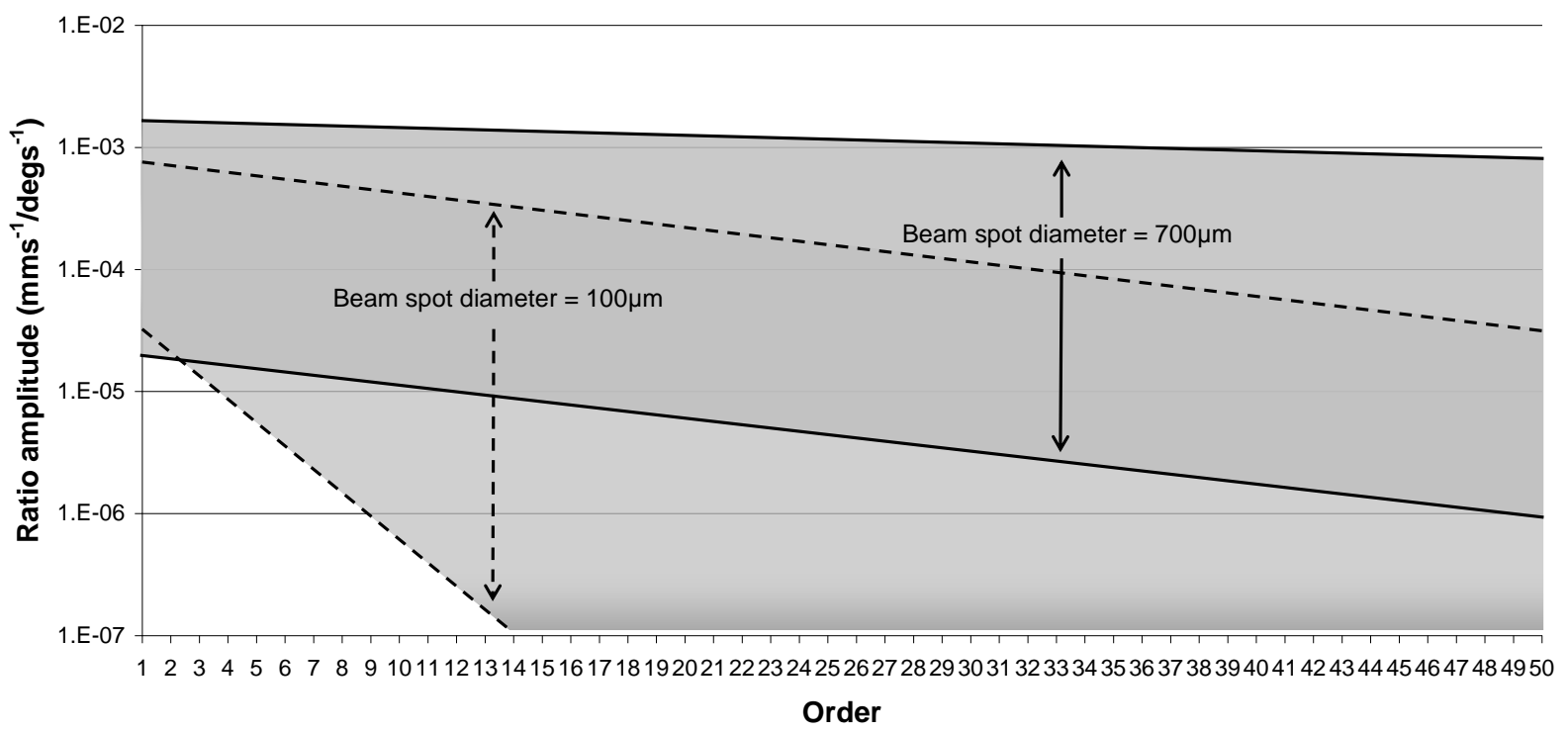

Figure 12 - Speckle noise normalised against the angular velocity at the fundamental frequency

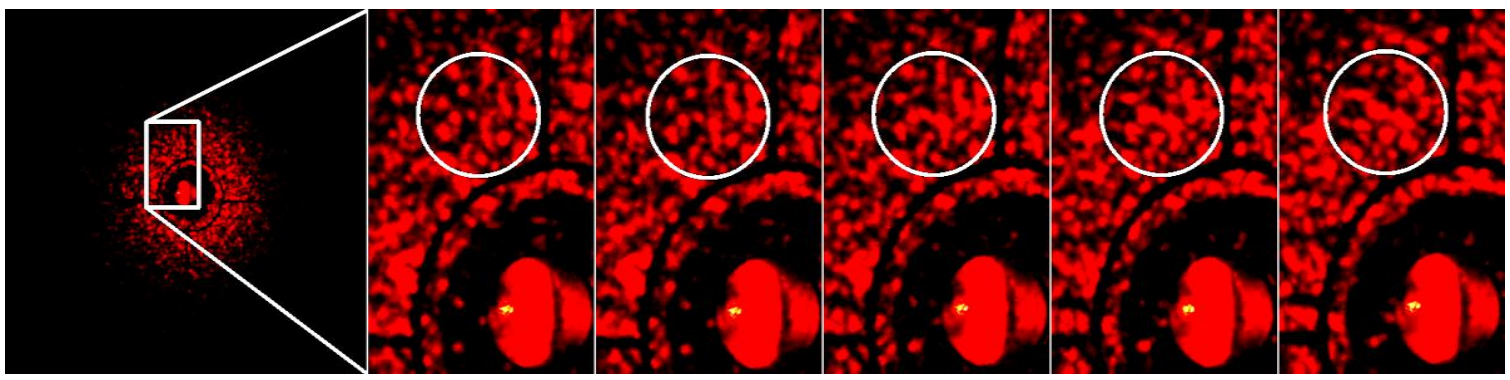

(a)

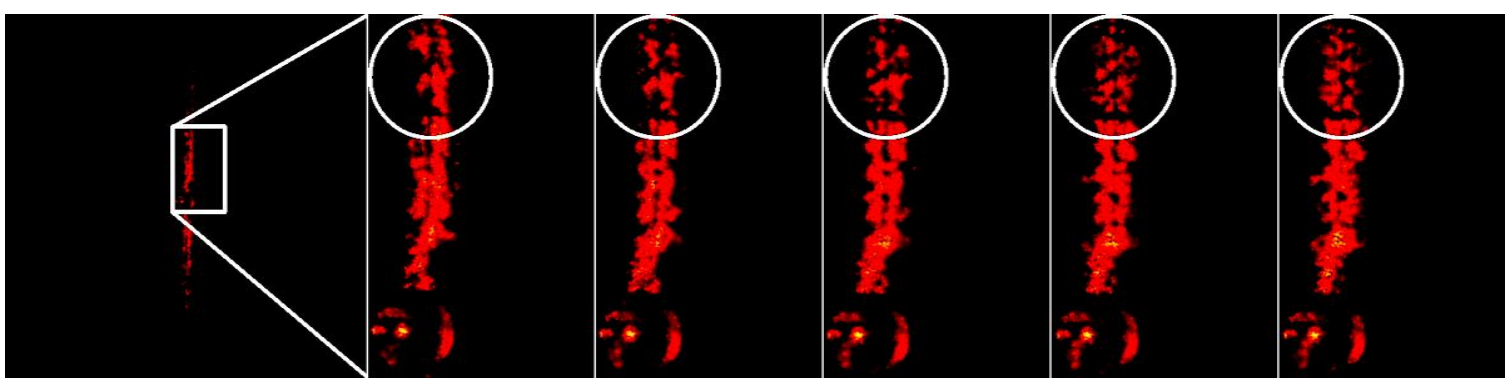

(b)

Figure 13 Speckle pattern and the motions from (a) retro-reflective tape, (b) metallic surface with roughness Ra $0.0618 \mu \mathrm{m}$ using a beam spot diameter $700 \mu \mathrm{m}$ 


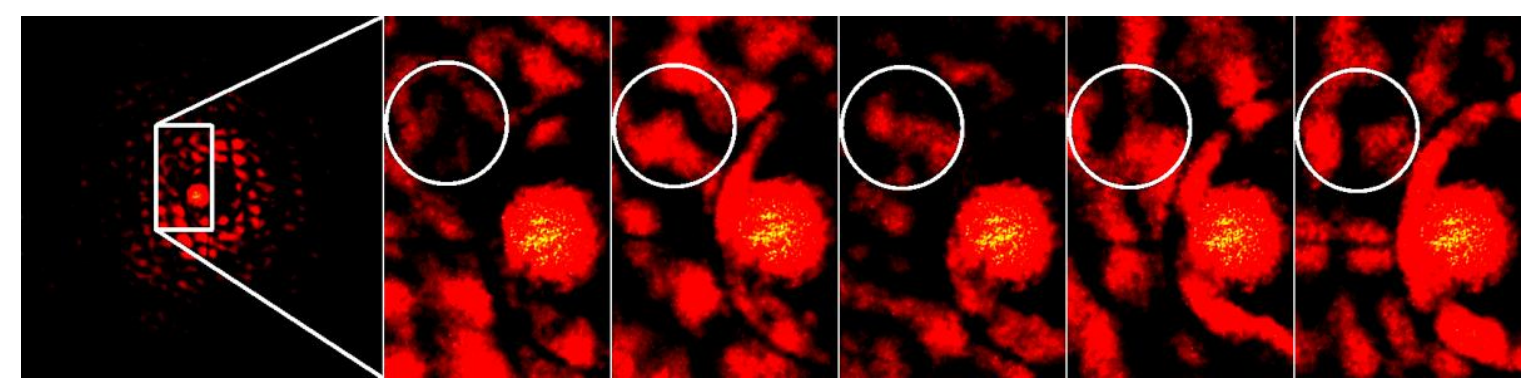

(a)
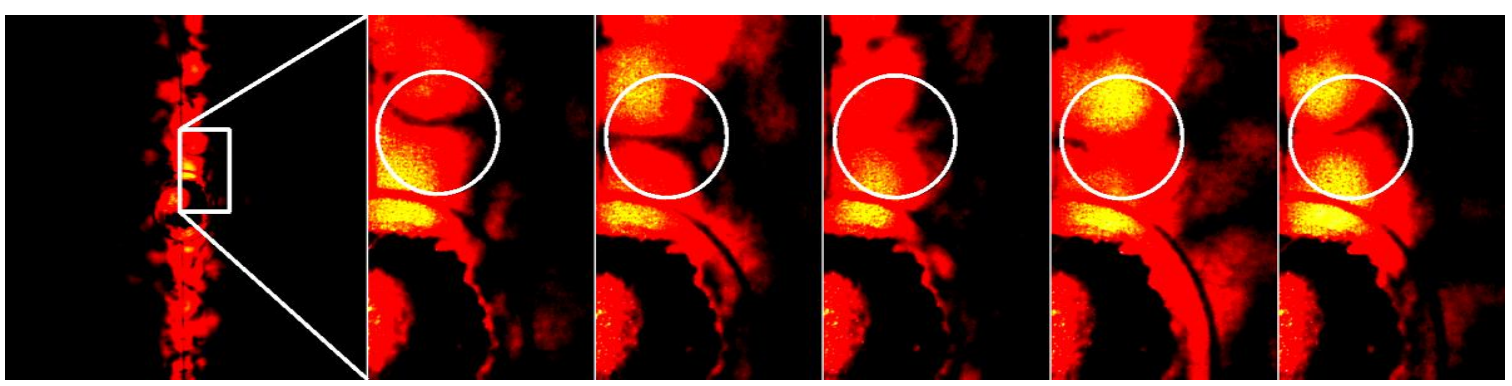

(b)

Figure 14 - Speckle pattern and the motions from (a) retro-reflective tape, (b) metallic surface with roughness Ra $0.0618 \mu \mathrm{m}$ using a beam spot diameter $100 \mu \mathrm{m}$

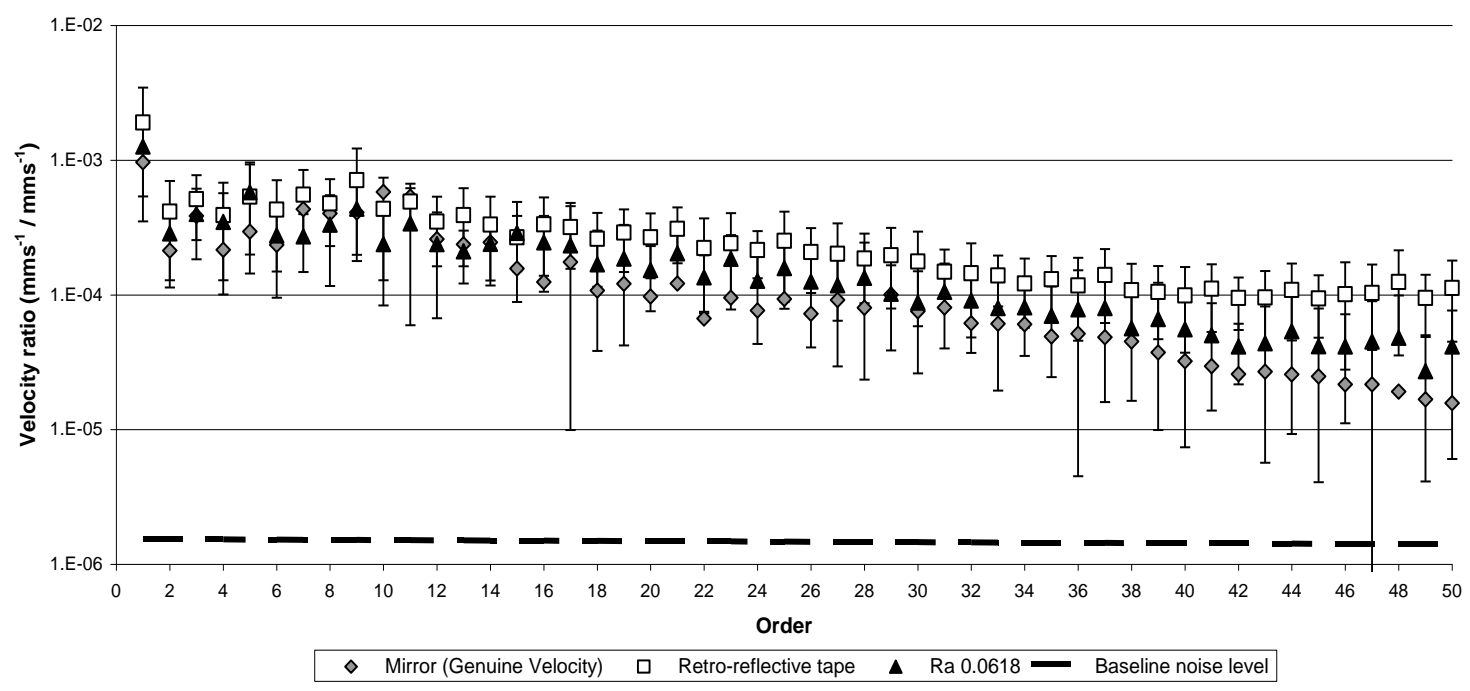

Figure 15- Measured velocity normalised against the transverse velocity at the fundamental frequency using a beam spot diameter $100 \mu \mathrm{m}$ 